\title{
The wave-corpuscle properties of microscopic particles in the nonlinear quantum-mechanical systems
}

\author{
Xiaofeng Pang \\ Institute of Life Science and Technology, University of Electronic Science and Technology of China, Chengdu 610054, China and \\ International Centre for Materials Physics, Chinese Academy of Science, Shenyang, China; \\ *Corresponding Author: pangxf2006@yahoo.com.cn
}

Received 12 December, 2010; revised 20 February, 2011; accepted 3 March 2011.

\begin{abstract}
We debate first the properties of quantum mechanics and its difficulties and the reasons resulting in these diffuculties and its direction of development. The fundamental principles of nonlinear quantum mechanics are proposed and established based on these shortcomings of quantum mechanics and real motions and interactions of microscopic particles and backgound field in physical systems. Subsequently, the motion laws and wave-corpuscle duality of microscopic particles described by nonlinear Schrödinger equation are studied completely in detail using these elementary principles and theories. Concretely speaking, we investigate the wave-particle duality of the solution of the nonlinear Schrödinger equation, the mechanism and rules of particle collision and the uncertainty relation of particle's momentum and position, and so on. We obtained that the microscopic particles obey the classical rules of collision of motion and satisfy the minimum uncertainty relation of position and momentum, etc. From these studies we see clearly that the moved rules and features of microscopic particle in nonlinear quantum mechanics is different from those in linear quantum mechanics. Therefore, nolinear quantum mechanics is a necessary result of development of quantum mechanics and represents correctly the properties of microscopic particles in nonlinear systems, which can solve difficulties and problems disputed for about a century by scientists in linear quantum mechanics field.
\end{abstract}

Keywords: Microscopic Particle; Nonlinear Interaction; Quantum Mechanics; Nonlinear Schrödinger Equation; Basic Principle; Nonlinear Theory; Wave-Particle Duality; Motion Rule

\section{INTRODUCTION, WAVE FEATURE OF MICROSCOPIC PARTICLES AND DIFFICULTIES OF QUANTUM MECHANICS}

It is well known that several great scientists, such as Bohr, Born, Schrödinger and Heisenberg, etc. established quantum mechanics in the early 1900s [1-9], which is the foundation and pillar of modern science and provides an unique way of describing the properties and rules of motion of microscopic particles (MIP) in microscopic systems. The elementary hypotheses of quantum mechanics can be described as Eq.1. The states of microscopic particles is described by a wave function $\phi(\boldsymbol{r}, t)$ or wave-vector, $|\phi(\boldsymbol{r}, t)\rangle$, which represents the state of the particle at position $r$ and time $t$ and satisfies the following superposition principle:

$$
\phi(\boldsymbol{r}, t)=c_{1} \phi_{1}(\boldsymbol{r}, t)+c_{2} \phi_{2}(\boldsymbol{r}, t) \text { or }|\phi\rangle=C_{1}\left|\phi_{1}\right\rangle+C_{2}\left|\phi_{2}\right\rangle
$$

where $\phi_{1}$ or $\left|\phi_{1}\right\rangle$ and $\phi_{2}$ or $\left|\phi_{2}\right\rangle$ are two states of the microscopic particle, $C_{1}$ and $C_{2}$ are constants relating to its states of a microscopic particle. The superposition principle manifests that the linear superposition of two different states of the particle describes still it's a state. Therefore, it is referred to as the linear superposition principle of states of the microscopic particle. The changed rules of the state of microscopic particle with varying of time and space satisfy the following Schrödinger equation:

$$
i \hbar \frac{\partial \phi}{\partial t}=-\frac{\hbar^{2}}{2 m} \nabla^{2} \phi+V(\boldsymbol{r}, t) \phi
$$

where $\hbar^{2} \nabla^{2} / 2 m$ is the kinetic energy operator, $V(\boldsymbol{r}, t)$ is the externally applied potential operator, $\mathrm{m}$ is the mass of particles, In this theory the Hamiltonian operator of the system corresponding dynamic Eq.2 is

$$
\hat{H}(t)=\hbar^{2} \nabla^{2} / 2 m+V(\boldsymbol{r}, t)
$$

(2) The mechanical quantity, which denotes the properties of microscopic particle, is represented by an op- 
erator. The value of a physical quantity $A$ in an arbitrary state $|\phi\rangle$ is given by some statistic average values, which are denoted by

$$
\langle\hat{A}\rangle=\langle\phi|\hat{A}| \phi\rangle /\langle\phi \mid \phi\rangle, \text { or }\langle\hat{A}\rangle=\langle\phi|\hat{A}| \phi\rangle \text {. }
$$

Only if the particle is in its eigenstate, then its mechanical quantities have determinant values. Thus a pair conjugate mechanical quantities cannot be simultaneously determined in a same state, i.e., their fluctuations satisfy the following Heisenberg uncertainty relation:

$$
\begin{aligned}
& \overline{(\Delta \hat{A})^{2}} \overline{(\Delta \hat{B})^{2}} \geq|\widehat{C}|^{2} / 4 \quad \text { with } i \widehat{C}=[\hat{A}, \widehat{B}] \\
& \text { and } \Delta A=\langle\hat{A}-\langle\hat{A}\rangle\rangle
\end{aligned}
$$

The quantum mechanics has achieved a great success in descriptions of motions of microscopic particles, such as, the electron, phonon, exciton, polaron, atom, molecule, atomic nucleus and elementary particles, and in predictions of properties of matter based on the motions of these particles. For example, energy spectra of atoms (such as hydrogen atom, helium atom), molecules (such as hydrogen molecules) and compounds, electrical, optical and magnetic properties of atoms and condensed matters can be calculated based on linear quantum mechanics and the calculated results are in basic agreement with experimental measurements. Thus considering that the quantum mechanics is thought of as the foundation of modern science, then the establishment of the theory of quantum mechanics has revolutionized not only physics, but also many other science branches such as chemistry, astronomy, biology, etc., and at the same time created many new branches of science, for instance, quantum statistics, quantum field theory, quantum electronics, quantum chemistry, quantum optics and quantum biology, etc. Therefore, we can say the quantum mechanics has achieved a great progress in modern science. One of the great successes of linear quantum mechanics is the explanation of the fine energy spectra of hydrogen atom, helium atom and hydrogen molecule. The energy spectra predicted by the quantum mechanics are in agreement with experimental data. Furthermore, new experiments have demonstrated that the results of the Lamb shift and superfine structure of hydrogen atom and the anomalous magnetic moment of the electron predicted by the theory of quantum electrodynamics are in agreement with experimental data. It is therefore believed that the quantum electrodynamics is one of the successful theories in modern physics [9-18]. Studying the above postulates in detail, we can find [7-13] that the quantum mechanics has the following characteristics.

1) Linearity. The wave function of the particles, $\psi(\boldsymbol{r}, t)$, satisfies the linear Schrödinger Eq.2 and linear superposition principle (1). In the meanwhile, the operators are some linear operators in the Hilbert space. This means that the quantum mechanics is a linear theory, thus it is quite reasonable to refer to the theory as the linear quantum mechanics.

2) The independence of Hamiltonian operator on the wave function. From Eq.3 we see clearly that the Hamiltonian operator of the systems is independent on the wave function of state of the particles, in which the interaction potential contained relates also not to the state of the particles. Thus the potential can change only the states of the particles, such as the amplitude, but not its natures. Therefore, the natures of the particles can only be determined by the kinetic energy term, $\hat{T}=\hbar^{2} \nabla^{2} / 2 m$ in Eqs. 2 and 3 .

3) Simplicity. We can easily solve arbitrary complicated quantum problems in the systems, only if their potential functions are obtained. Therefore, to solve quantum mechanical problems becomes almost to find the representations of the external potentials by means of various approximate methods. This theory states that once the externally applied potential field and initial states of the microscopic particles are given, the states of the particles at any time later and any position can be determined by the Schrödinger Eq.1 in the case of nonrelativistic motion.

4) The wave feature. The Schrödinger Eq.2 is in essence a wave equation and has only wave solutions, which do not include any corpuscle feature. In fact, let the wave function be $\phi=f \exp [-i E t / \hbar]$ and substitute it into Eq.2, we can obtain

$\partial^{2} f / \partial x^{2}+k_{0}^{2} n^{2} f=0$, where

$n^{2}=(E-U) /(E-C)=k^{2} / k_{0}^{2}, C$ is a constant,

$k_{0}^{2}=2 m(E-U) / \hbar^{2}$. This equation is nothing but that of a light wave propagating in a homogeneous medium. Thus, the linear Schrödinger Eq.2 is unique one able to describe the wave feature of the microscopic particle. In other words, when a particle moves continuously in the space-time, it follows the law of linear variation and disperses over the space-time in the form of a wave of microscopic particles. Therefore, the linear Schrödinger Eq.2 is a wave equation in essence, thus the microscopic particles are only a wave. This is a basic or essential nature of the microscopic particles in quantum mechanics.

This nature of the particles can be also verified by using the solutions of Eq.2 [7-18]. In fact, at $V(\boldsymbol{r}, t)=0$, its solution is a plane wave:

$$
\phi(\boldsymbol{r}, t)=A^{\prime} \exp [i(\boldsymbol{k} \cdot \boldsymbol{r}-\omega t)]
$$

where $k, \omega, A^{\prime}$ and are the wavevector, frequency, 
and amplitude of a wave, respectively. This solution denotes the state of a freely moving microscopic particle with an eigenenergy:

$$
E=\frac{p^{2}}{2 m}=\frac{1}{2 m}\left(p_{x}^{2}+p_{y}^{2}+p_{z}^{2}\right),\left(-\infty<p_{x}, p_{y}, p_{y}<\infty\right)
$$

This is a continuous spectrum. It states that the probability of the particle to appear at any point in the space is same, thus a microscopic particle propagates freely in a wave and distributes in total space, this means that the microscopic particle cannot be localized and has nothing about corpuscle feature.

If a free particle can be confined in a small finite space, such as, a rectangular box of dimension $a, b$ and $c$, the solution of Eq.1 is standing waves as follows:

$$
\phi(x, y, z, t)=A \sin \left(\frac{n_{1} \pi x}{a}\right) \sin \left(\frac{n_{2} \pi y}{b}\right) \sin \left(\frac{n_{3} \pi z}{c}\right) \mathrm{e}^{-i E t / \hbar}
$$

where $n_{1}, n_{2}$ and $n_{3}$ are three integers. In this case, the particle is still not localized, it appears also at each point in the box with a determinant probability. In this case the eigenenergy of the particle in this case is quantized as follows:

$$
E=\frac{\pi^{2} \hbar^{2}}{2 m}\left(\frac{n_{1}^{2}}{a^{2}}+\frac{n_{2}^{2}}{b^{2}}+\frac{n_{3}^{2}}{c^{2}}\right)
$$

where $n_{1}, n_{2}$ and $n_{3}$ are some integers. The corresponding momentum is also quantized. This means that the wave feature of microscopic particle has not been changed because of the variation of itself boundary condition.

If the potential field is further varied, for example, the microscopic particle is subject to a conservative time-independent field, $V(\boldsymbol{r}, t)=V(\boldsymbol{r}) \neq 0$, then the microscopic particle satisfies the time-independent linear Schrödinger equation

$$
-\frac{\hbar^{2}}{2 m} \nabla^{2} \phi^{\prime}+V(\boldsymbol{r}) \phi^{\prime}=E \phi^{\prime}
$$

where $\phi=\phi^{\prime}(\boldsymbol{r}) \mathrm{e}^{-i E t / \hbar}$. When $V=\boldsymbol{F} \cdot \boldsymbol{r}$, here $\boldsymbol{F}$ is a constant field force, such as, a one dimensional uniform electric field $\mathrm{E}^{\prime}$, then $V(x)=-\mathrm{e} E^{\prime} x$, thus its solution is

$$
\phi^{\prime}=A \sqrt{\bar{\xi}} H_{1 / 2}^{(1)}\left(\frac{2}{3} \bar{\xi}^{3 / 2}\right),\left(\bar{\xi}=\frac{x}{l}+\bar{\lambda}\right)
$$

where $H^{(1)}(x)$ is the first kind of Hankel function, $A$ is a normalized constant, $l$ is the characteristic length, and $\bar{\lambda}$ is a dimensionless quantity. The solution remains a dispersed wave. When $\xi \rightarrow \infty$, it approaches $\phi^{\prime}(\xi)=A^{\prime} \xi^{-1 / 4} \mathrm{e}^{-2 \xi^{3 / 2} / 3}$, which is a damped wave.

If $V(x)=a x^{2}$, the eigenenergy and eigenwave function are $\phi^{\prime}(x)=N_{n} e^{-a^{2} x^{2} / 2} H_{n}(\alpha x)$ with

$E_{n}=\left(n+\frac{1}{2}\right) \hbar \omega, \quad(n=0,1,2, \ldots)$, respectively, here $H_{n}(\alpha x)$ is the Hermite polynomial. The solution obviously has a decaying feature. If the potential fields are successively varied, we find that the wave nature of the solutions in Eq.2 does not change no matter what the forms of interaction potential. This shows clearly that the wave nature of the particles is intrinsic in quantum mechanics.

5) Quantization. The properties of microscopic particles are quantized in the microscopic systems. Concretely, the eigenvalues of physical quantities of the particles are quantized. For instance, the eigenenergy at $V(\boldsymbol{r}, t)=0$ is quantized as mentioned above, when $V(x)=a x^{2}$, its eigenenergy, $E_{n}=(n+1 / 2) \hbar \omega$, is also quantized, and so on. In practice, the momentum, moment of momentum and spin of the microscopic particles are all quantized in quantum mechanics. These quantized effects refer to as microscopic quantum effects, which occur on the microscopic scale.

Very sorry, the wave nature of the particles obtained from this theory is not only incompatible with de Broglie relation, $E=h v=\hbar \omega$ and $\boldsymbol{p}=\hbar \boldsymbol{k}$, of wave-corpuscle duality for microscopic particles and Davisson and Germer's experimental result of electron diffraction on double seam in 1927 [9-13], but also contradictory to the traditional concept of particles. Thus a lot of difficulties and problems occur in quantum mechanics, among them the central problem is how we represent the corpuscle feature of the microscopic particles. Aimed at this issue, Born introduce a statistic explanation for the wave function, and use $|\phi(\boldsymbol{r}, t)|^{2}$ to represent the probability of the particles occurring the position $\boldsymbol{r}$ at time $t$ in the space-time. However, the microscopic particles have a wave feature and can disperse over total system, thus the probability $|\phi(\boldsymbol{r}, t)|^{2}$ has a certain value at every point, for example, the probability of the particle denoted by Eq.5 is same at all points. This means that the particle can occur at every point at same time in the space. In this case, a fraction of particle must appear in the systems, which is a very strange phenomenon and is quite difficult to understand. However, in experiments, the particles are always captured as a whole one not a fractional one by a detector placed at an exact position. Therefore, the concept of probability representing the corpuscle behavior of the particles cannot be accepted [15-18].

On the other hand, we know from Eqs. 2 and 3 that the quantum mechanics requires to incorporate all interactions among particles or between particles and background field, such as the lattices in solids and nuclei in atoms and molecules, including nonlinear and complicated interactions, into the external potential by means of various approximate methods, such as, the free electron and average field approximations, Born-Oppenhei- 
mer approximation, Hartree-Fock approximation, Thomas Fermi approximation, and so on. This is obviously incorrect. The method replacing these real interactions by an average field amounts to freeze or blot out real motions and interactions of the microscopic particles and background fields, which was often used in the quantum mechanics to study the properties of the particles in the systems of many particles and many bodies [15-18]. This indicates that the quantum mechanics is only an approximate theory and therefore quantum mechanics cannot be used to solve the properties of the microscopic particles, such as electrons in atoms. In contrast, since the electron denoting by $\phi(\boldsymbol{r}, t)$ in atoms is a wave, then it does not have a determinant position in quantum mechanics, but the vector $\boldsymbol{r}$ is use to denote the position of the electron with charge $e$ and mass $m$ in the wave function and the Coulemb potential, $\mathrm{V}(\mathrm{r})=-\mathrm{Ze}^{2} / \boldsymbol{r}$. Thus it is difficult to understand correctly these contradictory representations in quantum mechanics.

These difficulties and problems of the quantum mechanics mentioned above inevitably evoked the contentions and further doubts about the theory among physicists. Actually, taking a closer look at the history of physics, we could find that not so many fundamental assumptions were required for a physical theory but the linear quantum mechanics. Obviously, these assumptions of linear quantum mechanics caused its incompleteness and limited its applicability. However, the disputations continued and expanded mainly between the group in Copenhagen School headed by Bohr representing the view of the main stream and other physicists, including Einstein, de Broglie, Schrödinger, Lorentz, etc. [7-18].

Why does quantum mechanics have these questions? This is worth studying deeply and in detail. As is known, dynamic Eq.2 describes the motion of a particle and Hamiltonian operator of the system, Eq.3, consist only of kinetic and potential operator of particles; the potential is only determined by an externally applied field, and not related to the state or wavefunction of the particle, thus the potential can only change the states of MIP, and cannot change its nature and essence. Therefore, the natures and features of MIP are only determined by the kinetic term. Thus there is no force or energy to obstruct and suppress the dispersing effect of kinetic energy in the system, then the MIP disperses and propagates in total space, and cannot be localized at all. This is the main reason why MIP has only wave feature in quantum mechanics. Meanwhile, the Hamiltonian in Eq.3 does not represent practical essences and features of MIP. In real physics, the energy operator of the systems and number operator of particles are always associated with the states of particles, i.e., they are related to the wavefunction of MIP. On the other hand, Eq.2 or 3 can de- scribe only the states and feature of a single particle, and cannot describe the states of many particles. However, a system composed of one particle does not exist in nature. The simplest system in nature is the hydrogen atom, but it consists of two particles. In such a case, when we study the states of particles in realistic systems composed of many particles and many bodies using quantum mechanics, we have to use a simplified and uniform average-potential unassociated with the states of particles to replace the complicated and nonlinear interaction among these particles [19-25]. This means that the motions of MIP and background field as well as the interactions between them are completely frozen in such a case. Thus, these complicated effects and nonlinear interactions determining essences and natures of particles are ignored completely, to use only a simplified or average potential replaces these complicated and nonlinear interactions. This is obviously not reasonable. Thus nature of MIP is determined by the kinetic energy term in Eq.2. Therefore, the microscopic particles described by quantum mechanics possess only a wave feature, not corpuscle feature. This is just the essence of quantum mechanics. Then we can only say that quantum mechanics is an approximate and linear theory and cannot represent completely the properties of motion of MIPs.

However, what is its direction of development? From the above studies we know that a key shortcoming or defect of LQM is its ignoring of dynamic states of other particles or background field and the dependence of the Hamiltonian or energy operator of the systems on the states of particles as well as nonlinear interactions among these particles. As a matter of fact, the nonlinear interactions always exist in any physics systems including the hydrogen atom, if only the real motions of the particles and background as well as their interactions are completely considered [17-30]. At the same time, it is also a reasonable assumption that the Hamiltonian or energy operator of the systems depend on the states of particles [19-32]. Hence, to establish a correct new quantum theory, we must break through the elementary hypotheses of LQM, and use the above reasonable assumptions to include the nonlinear interactions among the particles or between the particles and background field as well as the dependences of the Hamiltonian of the systems on the state of particles. Thus, we must establish nonlinear quantum mechanism (NLQM) to study the rules of motion of MIPs in realistic systems with nonlinear interactions by using the above method [19-32].

\section{ESTABLISHMENT OF NONLINEAR QUANTUM MECHANICS}

Pang worked out the NLQM describing the properties 
of motion of MIPs in nonlinear systems [17-30]. The elementary principles, theory, calculated rules and applications of NLQM are proposed and established based on the relations among the nonlinear interaction and soliton motions and macroscopic quantum effect through incorporating modern theories of superconductors, superfluids and solitons [23-27]. In these physical systems the Hamiltonian, free energy or Lagrangian functions of the systems are all nonlinear functions of the wave function of the microscopic particles which break down the hypotheses for the independence of the Hamiltonian of the systems on the states of the particles and the linearity of the theory in the LQM, the dynamic equations of microscopic particles, such as superconductive electrons and superfluid heliem atoms which were depicted by a macroscopic wave function, $\phi(\boldsymbol{r}, t)=\varphi(\boldsymbol{r}, t) \mathrm{e}^{i \theta(\boldsymbol{r}, t)}$, are the time-independent and time-dependent Ginzburg-Landau equations (G-L) and Gross-Pitaerskii (G-P) equation [33-38], which are in essence the nonlinear Schrödinger equation and have a soliton solution with a wave-corpuscle duality because the nonlinear interactions can balance and suppress the dispersive effect of the kinetic energy in these dynamic equations [23-27]. Therefore, the investigations of essences and properties of macroscopic quantum mechanics, superconductivity and superfluid provide direction for establishing nonlinear quantum mechanics [23-27].

Based on the above discussions, the fundamental principles of nonlinear quantum mechanics (NLQM) proposed by Pang can be summarized as follows [1932].

1) Microscopic particles are represented by the following wave function,

$$
\phi(\boldsymbol{r}, t)=\varphi(\boldsymbol{r}, t) \mathrm{e}^{i \theta(\boldsymbol{r}, t)}
$$

where both the amplitude $\varphi(\boldsymbol{r}, t)$ and phase $\theta(\boldsymbol{r}, t)$ of the wave function are functions of space and time, and satisfy different equation of motion.

2) In the nonrelativistic case, the wave function $\phi(\boldsymbol{r}, t)$ satisfies the generalized nonlinear Schrödinger equation (NLSE), i.e.,

$$
i \hbar \frac{\partial \phi}{\partial t}=-\frac{\hbar^{2}}{2 m} \nabla^{2} \phi \pm b|\phi|^{2} \phi+V(\boldsymbol{r}, t) \phi+A(\phi)
$$

or

$$
\mu \frac{\partial \varphi}{\partial t}=-\frac{\hbar^{2}}{2 m} \nabla^{2} \phi \pm b|\phi|^{2} \phi+V(\boldsymbol{r}, t) \phi+A(\phi)
$$

where $\mu$ is a complex number, $V$ is an external potential field, $A$ is a function of $\phi(\boldsymbol{r}, t)$, and $b$ is a coefficient indicating the strength of nonlinear interaction.

In the relativistic case, the wave function $\phi(r, t)$ satisfies the nonlinear Klein-Gordon equation (NLKGE), including the generalized Sine-Gordon equation (SGE) and the $\phi^{4}$-field equation, i.e.,

$$
\frac{\partial^{2} \phi}{\partial t^{2}}-\frac{\partial^{2} \phi}{\partial x_{j}^{2}}=\beta \sin \phi+\gamma \frac{\partial \phi}{\partial t}+A(\phi) \quad(\mathrm{j}=1,2,3)
$$

and

$$
\frac{\partial^{2} \phi}{\partial t^{2}}-\frac{\partial^{2} \phi}{\partial x_{j}^{2}} \mp \alpha \phi \pm \beta|\phi|^{2} \phi=A(\phi) \quad(\mathrm{j}=1,2,3)
$$

where $\gamma$ represents a dissipative or frictional effects, $\alpha$ is a constant, $\beta$ is a coefficient indicating the strength of nonlinear interaction and $\mathrm{A}$ is a function of $\phi(\boldsymbol{r}, t)$.

The Lagrange density function corresponding to Eq.8 at $A(\phi)=0$ is given by [23-27]:

$$
\begin{aligned}
\mathrm{L}^{\prime}= & \frac{\mathrm{i} \hbar}{2}\left(\phi^{*} \phi_{\mathrm{t}}-\phi \phi_{\mathrm{t}}^{*}\right)-\frac{\hbar^{2}}{2 \mathrm{~m}}\left(\nabla \phi \cdot \nabla \phi^{*}\right) \\
& -\mathrm{V}(\mathrm{x}) \phi^{*} \phi+(\mathrm{b} / 2)\left(\phi^{*} \phi\right)^{2}
\end{aligned}
$$

where $L^{\prime}=L$ is the Lagrange density function. The momentum density of the particle system is defined as $\mathrm{P}=\partial \mathcal{L} / \partial \phi$. Thus, the Hamiltonian density of the systems is as follows

$$
\begin{gathered}
H=\frac{\mathrm{i} \hbar}{2}\left(\phi^{*} \partial_{\mathrm{t}} \phi-\phi \partial_{\mathrm{t}} \phi^{*}\right)-L \\
=\frac{\hbar^{2}}{2 \mathrm{~m}}\left(\nabla \phi \cdot \nabla \phi^{*}\right)+V(\mathbf{x}) \phi \phi^{*}-(b / 2)\left(\phi \phi^{*}\right)^{2}
\end{gathered}
$$

where $H^{\prime}=H$ is the Hamiltonian density. Eqs.12 and 13 show clearly that the Lagrange density function and Hamiltonian density of the systems are all related to the wave function of state of the particles and involve a nonlinear interaction, $(b / 2)\left(\phi \phi^{*}\right)^{2}$. From the above fundamental principles, we see clearly that the NLQM breaks through the fundamental hypotheses of LQM in two aspects, namely the linearity of dynamic equations and independence of the Hamiltonian operator with the wave function of the particles. In the NLQM, the dynamic equations are all some nonlinear partial differential equations, in which nonlinear interactions, $b|\phi|^{2} \phi$, related to state wave function $\phi$ are involved; the Hamiltonian and Lagrangian operators in Eqs.12 and 13 corresponding to these equations also are all related to the state wave function $\phi$. Hence, so far as this point is concerned, the NLQM [23-27] is really a break-through or a new development in quantum mechanics. In nonlinear quantum mechanics the natures of microscopic particles are simultaneously determined by the kinetic and nonlinear interaction terms. Thus we expect [38-40] that the nonlinear interaction could suppress and balance the dispersive effect of kinetic energy of the particles in dynamics equations and make the particles be localized as soliton with wave-corpuscle feature. However, the nonlinear Schrödinger equation and nonlinear Klein- 
Gordon equation are evolved from linear Schrödinger equation and linear Klein-Gordon equation in linear quantum mechanics. Therefore, nonlinear quantum mechanics is a development of linear quantum mechanics. The superconductivity, superfluidity, macroscopic quantum effects of materials are the experimental foundation of nonlinear quantum mechanics, its theoretical basis is modern superconductive, superfluid and soliton theories [33-40], the mathematical foundation is the nonlinear partial differential equations and the soliton theory.

Based on the elementary principle Pang [23-27] established the theory of nonlinear quantum mechanics, which includes the superposition theorem of state of the particles, relation of nonlinear Fourier transformation, nonlinear perturbation theory, theory of nonlinear quantization, eigenvalue theory of nonlinear Schrödinger equation, calculated method of eigenenergy of Hamiltonian operator and relativistic theory of nonlinear quantum mechanics, collision and scattering theory of microscopic particles, and so on [25-27]. Thus a complete nonlinear quantum mechanics was established. Then we can investigate the rules and properties of motion of microscopic particles in any physical systems using these principle and theories of nonlinear quantum mechanics.

\section{THE WAVE-CORPUSCLE PROPERTIES OF MICROSCOPIC PARTICLES}

\subsection{Wave-Corpuscle Duality of Solution of Simple Nonlinear Schrödinger Equation}

As it is known, the microscopic particles have only the wave feature, but not corpuscle property in the quantum mechanics. Thus, it is very interesting what are the properties of the microscopic particles in the nonlinear quantum mechanics? We now study firstly the properties of the microscopic particles described by nonlinear Schrödinger equation in Eq.8. In the one-dimensional case, the Eq.8 at $\mathrm{V}(\mathrm{x}, \mathrm{t})=\mathrm{A}(\phi)=0$ becomes as

$$
i \phi_{t^{\prime}}+\phi_{x^{\prime} x^{\prime}}+b|\phi|^{2} \phi=0
$$

where $x^{\prime}=x / \sqrt{\hbar^{2} / 2 m}, t^{\prime}=t / \hbar$. We now assume the solution of Eq.11 to be of the form

$$
\phi\left(x^{\prime}, t^{\prime}\right)=\varphi\left(\xi^{\prime}\right) \mathrm{e}^{i k\left(x^{\prime}-x_{0}^{\prime}\right)-i \omega t^{\prime}}
$$

where $\xi^{\prime}=x^{\prime}-x_{0}^{\prime}-v_{e} t^{\prime}$. Inserting Eq.16 into Eq.15 we can obtain

$$
\varphi_{\xi^{\prime} \xi^{\prime}}\left(\xi^{\prime}\right)+i\left(2 k-v_{e}\right) \varphi_{\xi^{\prime}}+\left(\omega-k^{2}\right) \varphi\left(\xi^{\prime}\right)+b \varphi^{2} \varphi=0
$$

If the imaginary coefficient of $\varphi_{\xi^{\prime}}$ vanishes, then $k=v_{e} / 2$. Let $A=k^{2}-\omega$ we get from Eq.16

$$
\varphi_{\xi^{\prime} \xi^{\prime}}+b \varphi^{3}-A \varphi=0
$$

This equation can be integrated, which results in

$$
\left(\varphi_{\xi^{\prime}}\right)^{2}=D+A \varphi^{2}-b \varphi^{4} / 2
$$

where $D$ is an integral constant. The solution $\varphi\left(\xi^{\prime}\right)$ of Eq.18 is obtained by inverting an elliptic integral:

$$
\int_{\varphi_{0}}^{\varphi} \frac{\mathrm{d} \varphi}{\sqrt{D+A \varphi^{2}-b \varphi^{4} / 2}}= \pm \xi^{\prime}
$$

Let

$P\left(\varphi^{\prime}\right)=\left(\alpha_{1}-\varphi^{\prime 2}\right)\left(\varphi^{\prime 2}-\alpha_{2}\right)=-\varphi^{\prime 4}+A(2 / b)^{1 / 2} \varphi^{\prime 2}+D$, where $\varphi^{\prime}=(b / 2)^{1 / 4} \varphi$, from Eq.19 we can get $\left[K(k)-F\left(\varphi^{\prime}, k\right)\right]= \pm \xi^{\prime}$, where $K(k)$ and $F\left(\varphi^{\prime}, k\right)$ are the first associated elliptic integral and incomplete elliptic integral, respectively, and

$$
\begin{aligned}
& k=[\left.\left(\alpha_{1}-\alpha_{2}\right) / \alpha_{1}\right]^{-1 / 2}, \\
& \alpha_{1,2}=A /(2 b)^{1 / 2} \pm\left[D+\left(A^{2} / 2 b\right)\right]^{1 / 2} \text {. Using these and } \\
& \alpha=\varphi_{1,2}^{2}, \text { we have } \\
& \varphi^{\prime}\left[(b / 2)^{1 / 4} \xi^{\prime}\right]=\varphi_{1}^{\prime} \\
& \cdot\left\{1-\left[\left(1-\varphi_{1}^{\prime 2} / \varphi_{2}^{\prime 2}\right) \operatorname{sn}^{2}\left(\xi^{\prime}(b / 2)^{1 / 4}, k\right)\right]\right\}^{1 / 2}
\end{aligned}
$$

when

$$
D \rightarrow 0, \varphi_{1}^{\prime} \rightarrow \varphi_{0}^{\prime}, k \rightarrow 1, \varphi^{\prime} \rightarrow \varphi_{0}^{\prime} \sec h\left[\varphi_{0}^{\prime} \xi^{\prime}(b / 2)^{1 / 4}\right],
$$

where $\varphi_{0}^{\prime}=\left(2 A^{2} / b\right)^{1 / 4}$, the soliton solution of Eq.14 can be obtained and represented finally by

$$
\begin{aligned}
& \phi_{s}\left(x^{\prime}, t^{\prime}\right)=\sqrt{\frac{2 A}{b}} \\
& \cdot \sec h\left[\sqrt{A}\left(x^{\prime}-x_{0}^{\prime}-v_{e} t^{\prime}\right)\right] \exp \left\{i\left[k\left(x^{\prime}-x_{0}^{\prime}\right)-\omega t^{\prime}\right]\right\}
\end{aligned}
$$

Pang [19,23-32] represented eventually the solution of nonlinear Schrödinger equation in Eq.14 in the coordinate of $(x, t)$ by

$$
\phi(x, t)=A_{0} \sec h\left\{\frac{A_{0} \sqrt{b m}}{\hbar}\left[\left(x-x_{0}\right)-v t\right]\right\} \mathrm{e}^{i\left[m v\left(x-x_{0}\right)-E t\right] / \hbar}
$$

where $A_{0}=\sqrt{\left(m v^{2} / 2-E\right) / 2 b}, v$ is the velocity of motion of the particle, $E=\hbar \omega$. This solution is completely different from Eq.6, and consists of a envelop and carrier waves, the former is

$\varphi(x, t)=A_{0} \sec h\left\{A_{0} \sqrt{b m}\left[\left(x-x_{0}\right)-v t\right] / \hbar\right\}$ and a belltype non-topological soliton with an amplitude $\mathrm{A}_{0}$, the 
latter is the $\exp \left\{i\left[m v\left(x-x_{0}\right)-E t\right] / \hbar\right\}$. This solution is shown in Figure 1(a). Therefore, the particles described by nonlinear Schrödinger Eq.14 are solitons. The envelop $\varphi(x, t)$ is a slow varying function and is a mass centre of the particles; the position of the mass centre is just at $x_{0}, A_{0}$ is its amplitude, and its width is given by $W^{\prime}=2 \pi \hbar / A_{0} \sqrt{2 m}$. Thus, the size of the particle is $A_{0} W^{\prime}=2 \pi \hbar / \sqrt{2 m}$ and a constant. This shows that the particle has exactly a determinant size and is localized at $x_{0}$. Its form resemble a wave packet, but differ in essence from both the wave solution in Eq.6 and the wave packet mentioned above in linear quantum mechanics due to invariance of form and size in its propagation process. According to the soliton theory [39-40], the bell-type soliton in Eq.22 can move freely over macroscopic distances in a uniform velocity $v$ in space-time retaining its form, energy, momentum and other quasi-particle properties. However, the wave packet in linear quantum mechanics is not so and will be decaying and dispersing with increasing time. Just so, the vector $r$ or $x$ in the representation in Eq.22 has definitively a physical significance, and denotes exactly the positions of the particles at time $t$. Thus, the wavefunction $\phi(\boldsymbol{r}, t)$ or $\varphi(x, t)$ can represent exactly the states of the particle at the position $\vec{r}$ or $x$ at time $t$. These features are consistent with the concept of particles. Thus the microscopic particles depicted by Eq.14 display outright a corpuscle feature.

Using the inverse scattering method Zakharov and Shabat [41,42] obtained also the solution of Eq.14, which was represented as

$$
\begin{aligned}
& \varphi\left(x^{\prime}, t^{\prime}\right)=2\left(\frac{2}{b}\right)^{1 / 2} \eta \sec h\left[2 \eta\left(x^{\prime}-x_{0}^{\prime}\right)+8 \eta \xi t^{\prime}\right] \\
& \cdot \exp \left[-4 i\left(\xi^{2}-\eta^{2}\right) t^{\prime}-i 2 \xi x^{\prime}-i \theta\right]
\end{aligned}
$$

in the coordinate of $\left(x^{\prime}, t^{\prime}\right)$, where $\eta$ is related to the amplitude of the microscopic particle, $\xi$ relates to the velocity of the particle, $\theta=\arg \gamma, \lambda=\xi+i \eta$, $x_{0}^{\prime}=(2 \eta)^{-1} \log (|\gamma| / 2 \eta), \gamma$ is a constant. We now rewrite it as following form [23-29]:

$$
\phi\left(x^{\prime}, t^{\prime}\right)=2 \sqrt{\frac{2}{b}} k \sec h\left\{2 k\left[\left(x^{\prime}-x_{0}^{\prime}\right)-v_{e} t^{\prime}\right]\right\} \mathrm{e}^{i_{e}\left[\left(x^{\prime}-x^{\prime}\right)-v_{c} t^{\prime}\right] / 2}
$$

where $v_{\mathrm{e}}$ is the group velocity of the electron, $v_{c}$ is the phase speed of the carrier wave in the coordinate of $\left(x^{\prime}, t^{\prime}\right)$. For a certain system, $v_{e}$ and $v_{c}$ are determinant and do not change with time. We can obtain $2^{3 / 2} k / b^{1 / 2}=$ $A_{0}, A_{0}=\sqrt{\frac{v_{e}^{2}-2 v_{c} v_{e}}{2 b}}$. According to the soliton theory, the soliton in Eq.24 has determinant mass, momentum and energy, which are represented by [23-29]

$$
\begin{gathered}
N_{s}=\int_{-\infty}^{\infty}|\phi|^{2} \mathrm{~d} x^{\prime}=2 \sqrt{2} A_{0}, \\
p=-i \int_{-\infty}^{\infty}\left(\varphi^{*} \varphi_{x^{\prime}}-\varphi \varphi_{x^{\prime}}^{*}\right) \mathrm{d} x^{\prime}=2 \sqrt{2} A_{0} v_{e}, \\
=N_{s} v_{e}=\text { const } \\
E=\int_{-\infty}^{\infty}\left[\left|\phi_{x^{\prime}}\right|^{2}-\frac{1}{2}|\phi|^{4}\right] \mathrm{d} x^{\prime}=E_{0}+\frac{1}{2} M_{s o l} v_{e}^{2}
\end{gathered}
$$

where $M_{\text {sol }}=N_{s}=2 \sqrt{2} A_{0}$ is just effective mass of the particles, which is a constant. Thus we can confirm that the energy, mass and momentum of the particle cannot be dispersed in its motion, which embodies concretely the corpuscle features of the microscopic particles. This is completely consistent with the concept of classical particles. This means that the nonlinear interaction, $b|\phi|^{2} \phi$, related to the wave function of the particles, balances and suppresses really the dispersion effect of the kinetic term in Eq.14 to make the particles become eventually localized. Thus the position of the particles, $r$ or $x$, has a determinately physical significance.

However, the envelope of the solution in Eqs.22-24 is a solitary wave. It has a certain wave vector and frequency as shown in Figure 1(b), and can propagate in space-time, which is accompanied with the carrier wave. Its feature of propagation depends on the concrete nature of the particles. Figure 1(b) shows the width of the frequency spectrum of the envelope $\varphi(x, t)$ which has a localized distribution around the carrier frequency $\omega_{0}$. This shows that the particle has also a wave feature [23-29]. Thus we believe that the microscopic particles described by nonlinear quantum mechanics have simultaneously a wave-corpuscle duality. Eqs.22-24 and Figure 1(a) are just the most beautiful and perfect representation of this property, which consists also of de Broglie relation, $E=h v=\hbar \omega$ and $\boldsymbol{p}=\hbar \boldsymbol{k}$, wave-corpuscle duality and Davisson and Germer's experimental result of electron diffraction on double seam in 1927 as well as the traditional concept of particles in physics [11-15]. Thus we have reasons to believe the correctness of nonlinear quantum mechanics proposed by Pang.[23-29]

\subsection{Classical Natures of Collision of Microscopic Particles with Attractive Nonlinear Interactions}

\section{(1). The features of collision of microscopic parti-} cles

As a matter of fact, Zakharov and Shabat $[41,42]$ discussed firstly the properties of collision of two particles depicted by the nonlinear Schrödinger Eq.14 at $b=1>0$ and $b<0$. 


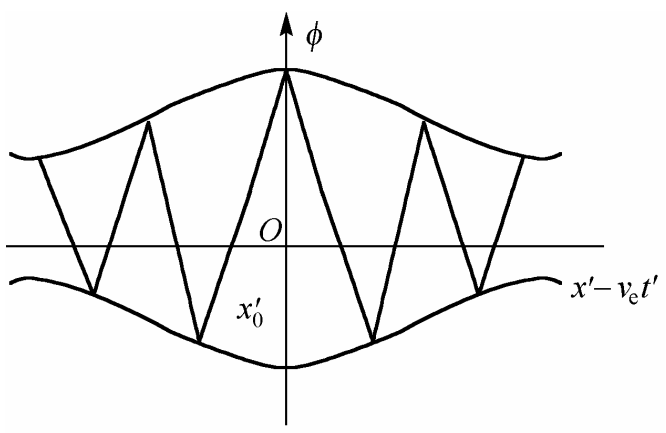

(a)

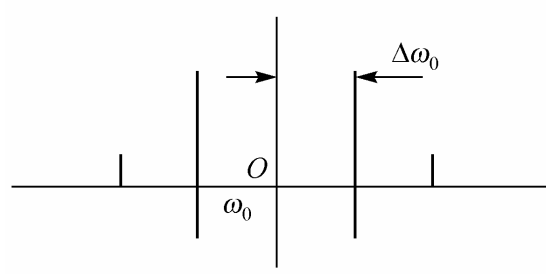

(b)

Figure 1. The solution of Eq.14 and its features.

According to Lax method [43], if two linear operators $\hat{L}$ and $\widehat{B}$ corresponding to Eq.14, which depend on $\phi$, satisfy the following Lax operator equation:

$$
i L_{t^{\prime}}=\hat{B} \hat{L}-\hat{L} \hat{B}=[\hat{B}, \hat{L}]
$$

where $\widehat{B}$ is a self-adjoint operator, then the eigenvalue $k$ and eigenfunction $\Psi$ of the operator $\hat{L}$ satisfy the equation:

$$
\hat{L} \Psi=k \Psi \text { with } \Psi\left(x^{\prime}, t^{\prime}\right)=\left(\begin{array}{c}
\Psi_{1} \\
\Psi_{2}
\end{array}\right),
$$

but $\hat{B}$ satisfies the equation: $\hat{B} \Psi=i \Psi_{t^{\prime}}$.

Zakharov and Shabat $[41,42]$ found out that the concrete representations of $\widehat{L}$ and $\widehat{B}$ for Eq.14, which are as follows

$$
\begin{aligned}
& \hat{L}=i\left(\begin{array}{cc}
1+s & 0 \\
0 & 1-s
\end{array}\right) \frac{\partial}{\partial x^{\prime}}+\left(\begin{array}{cc}
0 & \phi^{*} \\
\phi & 0
\end{array}\right) \\
& \hat{B}=-s\left(\begin{array}{ll}
1 & 0 \\
0 & 1
\end{array}\right) \frac{\partial^{2}}{\partial x^{\prime 2}}+\left(\begin{array}{cc}
\frac{|\phi|^{2}}{1+s} & i \phi_{x^{\prime}}^{*} \\
-i \phi_{x^{\prime}} & -\frac{|\phi|^{2}}{1-s}
\end{array}\right),
\end{aligned}
$$

where $s^{2}=(1-2 / b), \varphi(\boldsymbol{r}, t)$ satisfies Eq.14. We represent $\Psi$ in Eq.28 in one-dimensional case by

$$
\Psi=S \exp \left(-i \bar{k} x^{\prime}\right) \psi
$$

where $S=\left(\begin{array}{cc}0 & (1-s)^{1 / 2} \\ (1+s)^{1 / 2} & 0\end{array}\right), \quad \bar{k}=\frac{k}{1-s^{2}}=b k / 2$.
Inserting Eq.91 and $\hat{L}$ in Eq.90 into Eq.27 the Zakharov-Shabat (ZS) equation $[41,42]$ can be obtained as follows:

$$
\begin{aligned}
& \psi_{1 x^{\prime}}-q \psi_{2}=-i \lambda \psi_{1} \\
& \psi_{2 x^{\prime}}+q^{*} \psi_{1}=i \lambda \psi_{2}
\end{aligned}
$$

where $q=\frac{i \phi}{\left(1-s^{2}\right)^{1 / 2}}=i\left(\frac{b}{2}\right)^{\frac{1}{2}} \phi, \lambda=\bar{k} s$.

Zakharov and Shabat found out the soliton solution of Eq.14 using the inverse scattering method from ZS Eqs. 30 and 31, which is denoted in Eq.22, and studied further the properties of collision of these soliton solutions in these cases. In the studies of $b=1>0$, they gave first this single soliton solution of this equation, where $2 \sqrt{2} \eta$ is the amplitude of the soliton, $2 \sqrt{2} \xi$ denotes its velocity, $\lambda=\zeta=\xi+i \eta$ is the eigenvalues of the linear operator $\hat{L}$ in Eq.90, $x_{0}^{\prime}$ and $\theta^{+}$are the mass centre and phase of microscopic particle. In such a case they found further out the N-soliton solution of Eq.15 and studied thus the collision features of two solitons in the system. We here adopted their results of research to explain the rules and properties of collision between the microscopic particles in the nonlinear quantum mechanics. They $[41,42]$ find from calculation that the mass centre and phase of particle occur only change after this collision. The translations of the mass centre $x_{0 m}^{\prime+}$ and phase $\theta_{m}^{+}$of $m^{\text {th }}$ particles, which moves to a positive direction after this collision, can be represented, respectively, by

$$
\begin{aligned}
& x_{0 m}^{++}-x_{0 m}^{\prime}=\frac{1}{\eta_{m}} \prod_{p=m+1}^{N}\left|\frac{\lambda_{m}-\lambda_{p}}{\lambda_{m}-\lambda_{p}^{*}}\right|<0, \text { and } \\
& \theta_{m}^{+}-\theta_{m}=-2 \prod_{p=m+1}^{N} \arg \left(\frac{\lambda_{m}-\lambda_{p}}{\lambda_{m}-\lambda_{p}^{*}}\right)
\end{aligned}
$$

where $\eta_{m}$ and $\lambda_{m}$ are some constants related to the amplitude and eigenvalue of $\mathrm{m}^{\text {th }}$ particles, respectively. The equations show that shift of position of mass centre of the particles and their variation of phase are a constants after the collision of two particles moving with different velocities and amplitudes. The collision process of the two particles can be described from Eq.32 as follows. Before the collision and in the case of $t^{\prime} \rightarrow-\infty$ the slowest soliton is in the front while the fastest at the rear, they collide with each other at $t^{\prime}=0$, after the collision and $t^{\prime} \rightarrow \infty$, they are separated and the positions just reversed. Thus Zakharov and Shabat ${ }^{[41-42]}$ obtained that as the time $t$ varies from $-\infty$ to $\infty$, the relative change of mass centre of two particles, $\Delta x_{0 m}^{\prime}$, and their relative change of phases, $\Delta \theta_{m}$, can, respectively, de- 
noted as

$$
\begin{aligned}
& \Delta x_{0 m}^{\prime}=x_{0 m}^{++}-x_{0 m}^{\prime-} \\
& =\frac{1}{\eta_{m}}\left(\sum_{k=m+1}^{N} \ln \left|\frac{\lambda_{m}-\lambda_{p}}{\lambda_{m}-\lambda_{p}^{*}}\right|-\sum_{k=1}^{m-1} \ln \left|\frac{\lambda_{m}-\lambda_{p}}{\lambda_{m}-\lambda_{p}^{*}}\right|\right)
\end{aligned}
$$

and

$$
\begin{aligned}
\Delta \theta_{m} & =\theta_{m}^{+}-\theta_{m}^{-} \\
& =2 \prod_{k=1}^{m-1} \arg \left(\frac{\lambda_{m}-\lambda_{p}}{\lambda_{m}-\lambda_{p}^{*}}\right)-2 \prod_{k=m+1}^{N} \arg \left(\frac{\lambda_{m}-\lambda_{p}}{\lambda_{m}-\lambda_{p}^{*}}\right)
\end{aligned}
$$

where $x_{0 m}^{\prime-}$ and phase $\theta_{m}^{-}$are the mass centre and phase of $m^{\text {th }}$ particles at inverse direction or initial position, respectively. Eq.34 can be interpreted by assuming that the microscopic particles collide pair wise and every microscopic particle collides with others. In each paired collision, the faster microscopic particle moves forward by an amount of

$\eta_{m}^{-1} \ln \left|\left(\lambda_{m}-\lambda_{k}^{*}\right) / \lambda_{m}-\lambda_{k}\right|, \lambda_{m}>\lambda_{k}$, and the slower one shifts backwards by an amount of

$\eta_{k}^{-1} \ln \left|\left(\lambda_{m}-\lambda_{k}^{*}\right) / \lambda_{m}-\lambda_{k}\right|$. The total shift is equal to the algebraic sum of their shifts during the paired collisions. So that there is no effect of multi-particle collisions at all. In other word, in the collision process in each time the faster particle moves forward by an amount of phase shift, and the slower one shifts backwards by an amount of phase. The total shift of the particles is equal to the algebraic sum of those of the pair during the paired collisions. The situation is the same with the phases. This rule of collision of the microscopic particles described by the nonlinear Schrödinger Eq.14 is the same as that of classical particles, or speaking, meet also the collision law of macroscopic particles, i.e., during the collision these microscopic particles interact and exchange their positions in the space-time trajectory as if they had passed through each other. After the collision, the two microscopic particles may appear to be instantly translated in space and/or time but otherwise unaffected by their interaction. The translation is called a phase shift as mentioned above. In one dimension, this process results from two microscopic particles colliding head-on from opposite directions, or in one direction between two particles with different amplitudes or velocities. This is possible because the velocity of a particle depends on the amplitude. The two microscopic particles surviving a collision completely unscathed demonstrate clearly the corpuscle feature of the microscopic particles. This property separates the microscopic particles (solitons) described by the nonlinear quantum mechanics from the particles in the linear quantum mechanical regime. Thus this demonstrates the classical feature of the microscopic particles.

\section{(2). The results of numerical simulation of collision} of microscopic particles

Pang et al:[23-29,44] who further simulated numerically the collision behaviors of two particles described nonlinear Schrödinger Eq.8 at $V(x)=$ constant and $A(\phi)=0$ using the fourth-order Runge_Kutta method $^{[45-46]}$.

For the purpose we now divide Eq.8 at $A(\phi)=0$ and $b>0$ in one-dimensional case into the following two-equations

$$
\begin{gathered}
i \hbar \frac{\partial \phi}{\partial t}+\frac{\hbar^{2}}{2 m} \frac{\partial^{2} \phi}{\partial x^{2}}=\chi \phi \frac{\partial u}{\partial x}, \\
M\left(\frac{\partial^{2} u}{\partial t^{2}}-v_{0} \frac{\partial^{2} u}{\partial x^{2}}\right)=\chi \frac{\partial}{\partial x}\left(|\phi|^{2}\right) .
\end{gathered}
$$

Eqs.35 and 36 describe the features of motion of studied soliton and another particle ( such as, phonon) or background field (such as, lattice) with mass $M$ and velocity $v_{0}$, respectively, where $u$ is the characteristic quantity of another particle (as phonon) or of vibration (such as, displacement) of the background field. The coupling between the two modes of motion is caused by the deformation of the background field through the studied soliton - background field coupling, such as, dipole-dipole interaction, $\chi$ is the coupling coefficient between them and represents the change of interaction energy between the studied soliton and background field due to an unit variation of the background field. The relation between the two modes of motion can be obtained from Eq.36 and represented by

$$
\frac{\partial u}{\partial x}=\frac{\chi}{M\left(v^{2}-v_{0}^{2}\right)}|\phi|^{2}+A
$$

If inserting Eq.37 into Eq.35 yields just the nonlinear Schrödinger Eq.8 at V(x) = constant, where

$b=\frac{\chi^{2}}{M\left(v^{2}-v_{0}^{2}\right)}$ is a nonlinear coupling coefficient,

$V(x)=A \chi, \mathrm{A}$ is an integral constant. This result shows clearly that the nonlinear interaction $b|\phi|^{2} \phi$ comes from the coupling interaction between the studied soliton and background field. This is the reason what $b|\phi|^{2} \phi$ is referred to as nonlinear interaction.

In order to use fourth-order Runge-Kutta method ${ }^{[45-46]}$. to solve numerically Eqs.35 and $\mathbf{3 6}$ we must further discretize them, thus they are now denoted as

$$
\begin{aligned}
i \hbar \dot{\phi}_{n}(t) & =\varepsilon \phi_{n}(t)-J\left[\phi_{n+1}(t)+\phi_{n-1}(t)\right] \\
+ & \left(\chi / 2 r_{0}\right)\left[u_{n+1}(t)-u_{n-1}(t)\right] \phi_{n}(t) \\
M \ddot{u}_{n}(t) & =W\left[u_{n+1}(t)-2 u_{n}(t)+u_{n-1}(t)\right] \\
+ & \left(\chi / 2 r_{0}\right)\left[\left|\phi_{n+1}\right|^{2}-\left|\phi_{n-1}\right|^{2}\right]
\end{aligned}
$$


where $\dot{\phi}_{n}(t)=\frac{\partial \phi_{n}(t)}{\partial t}, \ddot{u}_{n}(t)=\frac{\partial^{2} u_{n}(t)}{\partial t^{2}}$ and the following transformation relation between continuous and discrete functions are used

$$
\begin{gathered}
\phi(x, t) \rightarrow \phi_{n}(t) \text { and } u(x, t) \rightarrow u_{n}(t), \\
\phi_{n \pm 1}(t)=\phi_{n}(t) \pm r_{0} \frac{\partial \phi_{n}(t)}{\partial x}+\frac{1}{2 !} r_{0}^{2} \frac{\partial^{2} \phi_{n}(t)}{\partial x^{2}} \pm \cdots \\
u_{n \pm 1}(t)=u_{n}(t) \pm r_{0} \frac{\partial u_{n}(t)}{\partial x}+\frac{1}{2 !} r_{0}^{2} \frac{\partial^{2} u_{n}(t)}{\partial x^{2}} \pm \cdots
\end{gathered}
$$

where $\varepsilon=\hbar^{2} / m r_{0}+A \chi, J=\hbar^{2} / 2 m r_{0}^{2}, W=M v_{0}^{2} / r_{0}^{2}$ $r_{0}$ is distance between neighboring two lattice points. If using transformation: $\phi_{n} \rightarrow \phi_{n} \exp (i \varepsilon t / \hbar)$ we can eliminate the term $\varepsilon \phi_{n}(t)$ in Eq.38. Again making a transformation: $\phi_{n}(t) \rightarrow a_{n}(t)=a(t) r_{n}+i a(t) i_{n}$, then Eqs.38 and $\mathbf{3 9}$ become

$$
\begin{gathered}
\hbar \dot{a} r_{n}=-J\left(a i_{n+1}+a i_{n-1}\right)+\left(\chi / 2 r_{0}\right)\left(u_{n+1}-u_{n-1}\right) a i_{n} \\
\hbar \dot{a} i_{n}=-J\left(a r_{n+1}+a r_{n-1}\right)+\left(\chi / 2 r_{0}\right)\left(u_{n+1}-u_{n-1}\right) a r_{n} \\
\dot{u}_{n}=y_{n} / M \\
\dot{y}_{n}=W\left(u_{n+1}-2 u_{n}+u_{n-1}\right) \\
+\left(\chi / 2 r_{0}\right)\left(a r_{n+1}^{2}+a i_{n+1}^{2}-a r_{n-1}^{2}-a i_{n-1}^{2}\right) \\
\left|a_{n}\right|^{2}=\left|a r_{n}\right|^{2}+\left|a i_{n}\right|^{2}=\left|\phi_{n}\right|^{2}
\end{gathered}
$$

where $a r_{n}$ and $a i_{n}$ are real and imaginary parts of $\mathrm{a}_{\mathrm{n}}$. Eqs.41-45 can determine states and behaviors of the microscopic particle. Their solutions can be found out from the four equations. There are four equations for one structure unit. Therefore, for the quantum systems constructed by $\mathrm{N}$ structure units there are $4 \mathrm{~N}$ associated equations. When the fourth-order Runge-Kutta method $[45,46]$ is used to numerically calculate these solutions we must further discretize them, in which $\mathrm{n}$ is replaced by $j$ and let the time be denoted by $n$, the step length of the space variable is denoted by $h$ in the above equations. An initial excitation is required in this calculation, which is chosen as, $a_{n}(\mathrm{o})=A \operatorname{Sech}\left[\left(n-n_{0}\right) \quad\left(\chi / 2 r_{0}\right)^{2} / 4 \mathrm{JW}\right]$ (where $A$ is the normalization constant) at the size $\mathrm{n}$, for the applied lattice, $u_{n}(0)=y_{n}(0)=0$. In the numerical simulation it is required that the total energy and the norm (or particle number) of the system must be conserved. The system of units, ev for energy, A for length and ps for time are proven to be suitable for the numerical solutions of Eqs.41-44. The one dimensional system is composed of $N$ units and fixed, where $\mathrm{N}$ is chosen to be $N=200$, and a time step size of 0.0195 is used in the simulations. Total numerical simulation is performed through data parallel algorithms and MALAB language.

If the values of the parameters, $M, \varepsilon, J, W, \chi$ and $r_{0}$ in Eqs.38 and $\mathbf{3 9}$ are appropriately chosen we can calculate the numerical solution of the associated Eqs.41-44 by using the fourth-order Runge-Kutta method $[45,46]$, thus the changes of $\left|\phi_{n}(t)\right|^{2}=\left|a_{n}(t)\right|^{2}$, which is probability or number density of the particle occurring at the nth structure unit, with increasing time and position in time-place can be obtained. This result is shown in Figure 3, which shows that the amplitude of the solution can retain constancy in motion process, i.e., the solution of Eqs.38 and $\mathbf{3 9}$ or Eq.8 at $V(x)=$ constant is very stable while in motion. In the meanwhile, we give the propagation feature of the solutions of Eqs.4144 in the cases of a long time period of 250ps and long spacings of 400 in Figure 3, which indicates that the states of solution are also stable in the long propagation. According to the soliton theory $[39,40]$ we can obtain that Eqs.38 and 39 have exactly a soliton solution, which have a feature of classical particles.

In order to verify the corpuscle feature of the solution of nonlinear Schrödinger Eq.8 we study their collision property in accordance with the soliton theory ${ }^{[39-40]}$. Thus we further simulated numerically the collision behaviors of two solitonn solutions of Eq.8 at

$V(x)=\varepsilon=\hbar^{2} / m r_{0}+A \chi=$ constant using the fourth-order Runge-Kutta method ${ }^{[45-46]}$. This process resulting from two particles colliding head-on from opposite directions, which are set up from opposite ends of the channel, is shown Figure 4, where the above initial conditions simultaneously motivate the opposite ends of the channels. From this figure we see clearly that the initial two particles having clock shapes and separating 50 unit spacings in the channel collide with each other at about $8 \mathrm{ps}$ and 25 units. After this collision, the two solitons in the channel go through each other without scattering obtained by Zakharov and Shabat $[41,42]$ as mentioned above. Clearly, the property of collision of the and retain their clock shapes to propagate toward and separately along itself channels. The collision properties of the solitons described by the nonlinear Schrödinger Eq.8 are same with those solutions of Eq.8 is same with the rules of collision of macroscopic particles. Thus, we can conclude that microscopic particles described by nonlinear Schrödinger Eq.8 have a corpuscle feature.

However, we see clearly that there is a wave peak with large amplitude in the colliding process in Figure 4. Obviously, this is a result of complicated superposition of solitary waves of two particles. This result displays the wave feature of the particles. Therefore, the collision process shown in Figure 3 represent obviously that the soliton solutions of the nonlinear Schrödinger equation have a both corpuscle and wave feature, which is due to the nonlinear interaction $b|\phi|^{2} \phi$. 
One words, the above properties of propagation and collision of particles described by the nonlinear Schrödinger equation with an external applied potential show that the particles are stable in propagation, and they can go through each other retaining their form after the collision of head-on from opposite directions, This feature is the same with that of the classical particles.
However, a wave peak with large amplitude, which is a result of complicated superposition of two solitary waves, occur in the colliding process. This displays the wave feature of the solitons. Therefore, the collision property of the solitons shows clearly that the solutions of the nonlinear Schrödinger equation have a both corpuscle and wave feature. Obviously, this is due to the nonlinear

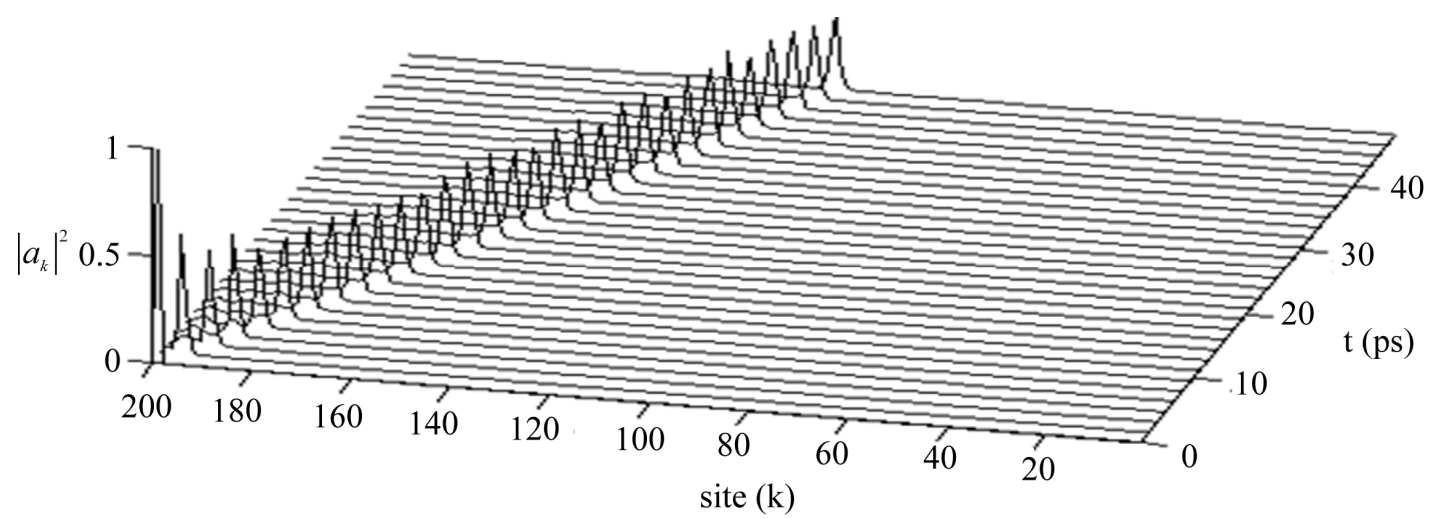

Figure 2. Motion of soliton solution of Eqs.38 and 39.

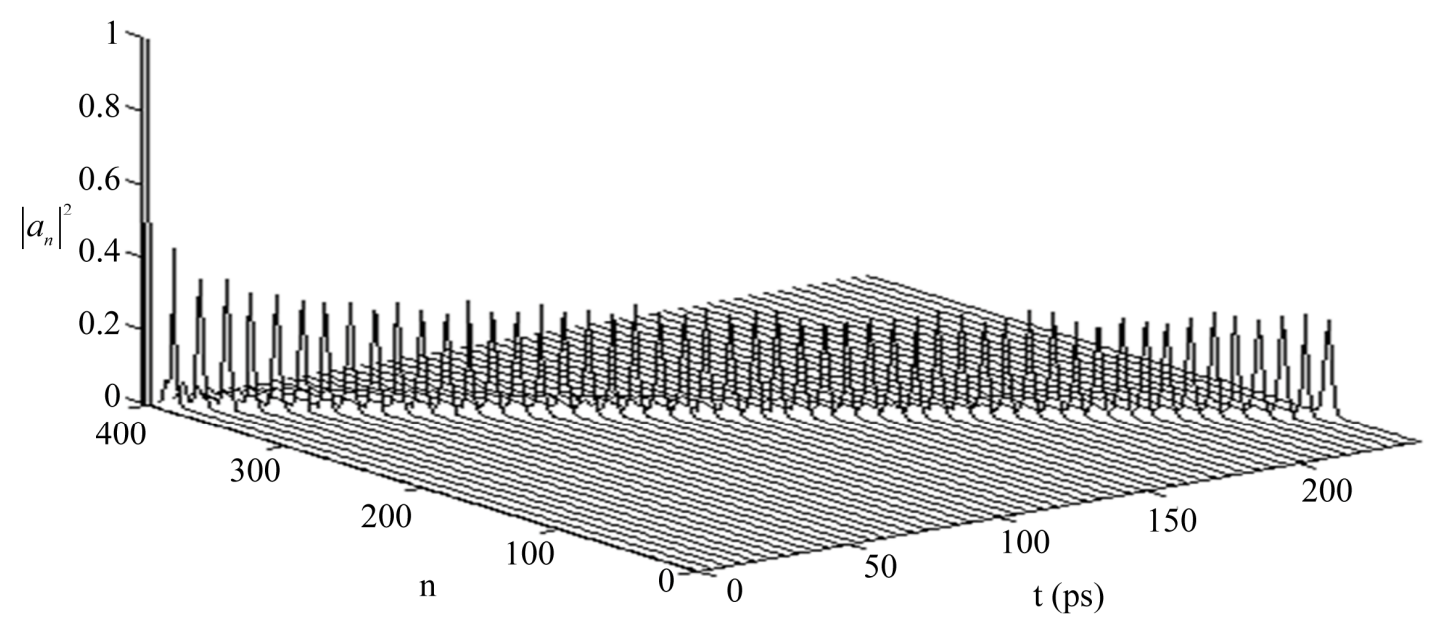

Figure 3. State of motion of microscopic particle described by Eqs.38 and $\mathbf{3 9}$ in the cases of a long time period and long spacings.

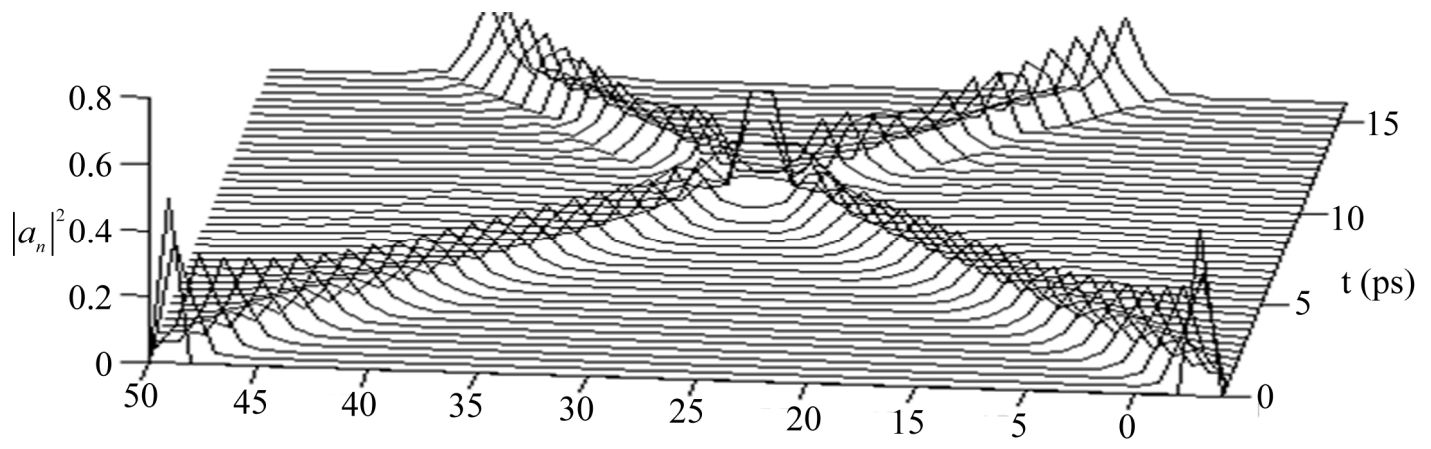

Figure 4. the features of collision of microscopic particles. 
interaction $b|\phi|^{2} \phi$, which suppresses the dispersive effect of kinetic energy in the dynamic equation. Thus the microscopic particles have a wave-corpuscle duality in this case.

\subsection{The Uncertainty Relationship for the Position and Momentum}

(1). Correct form of uncertainty relation in the linear quantum mechanics

As it is known, the microscopic particle has not a determinant position, disperses always in total space in a wave form in the linear quantum mechanics. Hence, the position and momentum of the microscopic particles cannot be simultaneously determined. This is just the well-known uncertainty relation. The uncertainty relation is an important formulae and also an important problem in the linear quantum mechanics that troubled many scientists. Whether this is an intrinsic property of microscopic particle or an artifact of the linear quantum mechanics or measuring instruments has been a longlasting controversy. Obviously, it is closely related to elementary features of microscopic particles. Since we have established the nonlinear quantum mechanics, in which the natures of the microscopic particles occur considerable variations relative to that in the linear quantum mechanics, thus we expect that the uncertainty relation in nonlinear quantum mechanics could be changed relative to that in the linear quantum mechanics. Then the significance and essence of the uncertainty relation can be revealed by comparing the results of linear and nonlinear quantum theories.

The uncertainty relation in the linear quantum mechanics can be obtained from $[25-29,47]$

$$
\mathrm{I}\left(\zeta^{\prime}\right)=\int\left|\left(\zeta^{\prime} \Delta \hat{\mathrm{A}}+\mathrm{i} \Delta \hat{\mathrm{B}}\right) \phi(\boldsymbol{r}, \mathrm{t})\right|^{2} \mathrm{~d} \boldsymbol{r} \geq 0
$$

or

$$
\overline{\hat{\mathrm{F}}}\left(\zeta^{\prime}\right)=\int \phi^{*}(\boldsymbol{r}, \mathrm{t}) \overrightarrow{\mathrm{F}}[\hat{\mathrm{A}}(\boldsymbol{r}, \mathrm{t}), \hat{\mathrm{B}}(\boldsymbol{r}, \mathrm{t})] \phi(\boldsymbol{r}, \mathrm{t}) \mathrm{d} \boldsymbol{r}
$$

In the coordinate representation, $\hat{A}$ and $\hat{B}$ are operators of two physical quantities, for example, position and momentum, or energy and time, and satisfy the commutation relation $[\hat{A}, \hat{B}]=i \hat{C}, \phi(\boldsymbol{r}, \mathrm{t})$ and $\phi^{*}(\boldsymbol{r}, \mathrm{t})$ are wave functions of the microscopic particle satisfying the Schrödinger equation 1.7 and its conjugate equation, respectively, $\hat{\mathrm{F}}=\left(\Delta \widehat{\mathrm{A}} \zeta^{\prime}+\Delta \widehat{\mathrm{B}}\right)^{2}$,

$(\Delta \hat{\mathrm{A}}=\hat{\mathrm{A}}-\overline{\mathrm{A}}, \Delta \hat{\mathrm{B}}=\hat{\mathrm{B}}-\overline{\mathrm{B}}, \overline{\mathrm{A}}$ and $\overline{\mathrm{B}}$ are the average values of the physical quantities in the state denoted by $\phi(r, \mathrm{t}))$, is an operator of physical quantity related to $\overline{\mathrm{A}}$ and $\overline{\mathrm{B}}, \xi$ is a real parameter. After some simplifications, we can get from Eq.46

$$
\begin{aligned}
& \mathrm{I}=\overline{\mathrm{F}}=\overline{\Delta \hat{\mathrm{A}}^{2}} \zeta^{\prime 2}+2 \overline{\Delta \hat{\mathrm{A}} \Delta \hat{\mathrm{B}}} \zeta^{\prime}+\overline{\Delta \hat{\mathrm{B}}^{2}} \geq 0 \text { or } \\
& \overline{\Delta \hat{\mathrm{A}}^{2}} \zeta^{\prime 2}+\overline{\hat{\mathrm{C}}} \zeta^{\prime}+\overline{\Delta \hat{\mathrm{B}}^{2}} \geq 0
\end{aligned}
$$

Using mathematical identities, this can be written as

$$
\overline{\Delta \hat{\mathrm{A}}^{2}} \overline{\Delta \hat{\mathrm{B}}^{2}} \geq \overline{\hat{\mathrm{C}}}^{2} / 4
$$

This is the uncertainty relation which is often used in the linear quantum mechanics. From the above derivation we see that the uncertainty relation was obtained based on the fundamental hypotheses of the linear quantum mechanics, including properties of operators of the mechanical quantities, the state of particle represented by the wave function, which satisfies the Schrödinger Eq.2, the concept of average values of mechanical quantities and the commutation relations and eigenequation of operators. Therefore, we can conclude that the uncertainty relation in Eq.48 is a necessary result of the quantum mechanics. Since the linear quantum mechanics only describes the wave nature of microscopic particles, the uncertainty relation is a result of the wave feature of microscopic particles, and it inherits the wave nature of microscopic particles. This is why its coordinate and momentum cannot be determined simultaneously. This is an essential interpretation for the uncertainty relation Eq.48 in the linear quantum mechanics. It is not related to measurement, but closely related to the linear quantum mechanics. In other words, if the linear quantum mechanics could correctly describe the states of microscopic particles, then the uncertainty relation should also reflect the peculiarities of microscopic particles.

Eq.48 can be written in the following form [25-29,47]:

$$
\begin{aligned}
\hat{\mathrm{F}} & =\overline{\Delta \hat{\mathrm{A}}^{2}}\left(\zeta \overline{\Delta \hat{\mathrm{A}} \Delta \hat{\mathrm{B}}} / \Delta \overline{\hat{\mathrm{A}}^{2}}\right)^{2} \\
& +\overline{\Delta \hat{\mathrm{B}}^{2}}-(\overline{\Delta \hat{\mathrm{A}} \Delta \hat{\mathrm{B}}})^{2} / \overline{\Delta \hat{\mathrm{A}}^{2}} \geq 0
\end{aligned}
$$

or

$$
\overline{\Delta \hat{\mathrm{A}}^{2}}\left(\zeta^{\prime}+\overline{\hat{C}} / 4 \overline{\Delta \hat{\mathrm{A}}^{2}}\right)^{2}+\overline{\Delta \hat{\mathrm{B}}^{2}}-(\overline{\hat{C}})^{2} / 4 \overline{\Delta \hat{\mathrm{A}}^{2}} \geq 0
$$

This shows that $\overline{\Delta \hat{\mathrm{A}}^{2}} \neq 0$, if $(\overline{\Delta \hat{\mathrm{A}} \Delta \hat{\mathrm{B}}})^{2}$ or $\overline{\hat{C}}^{2} / 4$ is not zero, else, we cannot obtain Eq.48 and

$$
\overline{\Delta \hat{\mathrm{A}}^{2}} \overline{\Delta \hat{B}^{2}}>\overline{(\Delta \hat{A} \Delta \hat{B})^{2}} \text { because when } \overline{\Delta \hat{A}^{2}}=0 \text {, Eq.50 }
$$

does not hold. Therefore, $\overline{\left(\Delta \hat{A}^{2}\right)} \neq 0$ is a necessary condition for the uncertainty relation Eq.48, $\Delta \hat{A}^{2}$ can approach zero, but cannot be equal to zero. Therefore, in the linear quantum mechanics, the right uncertainty relation should take the form:

$$
\overline{\Delta \hat{A}^{2} \Delta \hat{B}^{2}}>\left(\overline{\hat{C}}^{2}\right)^{2} / 4
$$




\section{(2). Uncertainty relation of microscopic particle in nonlinear quantum mechanics}

We now return to study the uncertainty relation of the microscopic particles described by the nonlinear quantum mechanics. In such a case the microscopic particles is a soliton and have a wave-corpuscle duality. Thus we have the reasons to believe that the uncertainty relation in this case should be different from equation (115) in the linear quantum theory.

Pang $[26-29,67]$ derived this relation for position and momentum of a microscopic particle depicted by the nonlinear Schrödinger Eq.14 with a solution, $\phi_{s}$, as given in Eq.22. The function $\phi_{s}\left(x^{\prime}, t^{\prime}\right)$ is a square integral function localized at $x_{0}^{\prime} \neq 0$ in the coordinate space. The Fourier transform of this function is given by

$$
\phi_{s}\left(p^{\prime}, t^{\prime}\right)=\frac{1}{\sqrt{2 \pi}} \int_{-\infty}^{\infty} \phi_{s}\left(x^{\prime}, t^{\prime}\right) \mathrm{e}^{-i p^{\prime} x^{\prime}} \mathrm{d} x^{\prime}
$$

Using Eq.22, then the Fourier transform is explicitly represented as

$$
\begin{aligned}
& \phi_{s}\left(p, t^{\prime}\right)=\sqrt{\frac{\pi}{2}} \operatorname{sech}\left[\frac{\pi}{4 \sqrt{2} \eta}\left(p^{\prime}-2 \sqrt{2} \xi\right)\right] \\
& \left.\cdot \exp \left\{i 4\left(\eta^{2}+\xi^{2}-p^{\prime} \xi / 2 \sqrt{2}\right) t^{\prime}-i\left(p^{\prime}-2 \sqrt{2} \xi\right) x_{0}^{\prime}+i \theta\right)\right\}
\end{aligned}
$$

It shows that $\phi_{s}\left(p^{\prime}, t^{\prime}\right)$ is also localized at $\mathrm{p}$ in momentum space. Eqs.22 and $\mathbf{5 3}$ show that the microscopic particle is localized in the shape of soliton not only in position space but also in the momentum space. For convenience, we introduce the normalization coefficient $B_{0}$ in Eqs.22 and 53, then obviously $B_{o}^{2}=\eta / 4 \sqrt{2}$, the position of the mass center of the microscopic particle, $\left\langle x^{\prime}\right\rangle$, and its square,

$\left\langle x^{\prime 2}\right\rangle$, at $t^{\prime}=0$ are given by

$$
\left\langle x^{\prime}\right\rangle=\int_{-\infty}^{\infty} x^{\prime}\left|\phi_{s}\left(x^{\prime}\right)\right|^{2} \mathrm{~d} x^{\prime},\left\langle x^{\prime 2}\right\rangle=\int_{-\infty}^{\infty} x^{\prime 2}\left|\phi_{s}\left(x^{\prime}\right)\right|^{2} \mathrm{~d} x^{\prime} .
$$

We can thus find that

$$
\left\langle x^{\prime}\right\rangle=4 \sqrt{2} \eta A_{0}^{2} x_{0}^{\prime},\left\langle x^{\prime 2}\right\rangle=\frac{A_{0}^{2} \pi^{2}}{12 \sqrt{2} \eta}+4 \sqrt{2} A_{0}^{2} \eta x_{0}^{\prime 2}
$$

respectively. Similarly, the momentum of the mass center of the microscopic particle, $\left\langle p^{\prime}\right\rangle$, and its square, $\left\langle p^{\prime 2}\right\rangle$, are given by

$$
\left\langle p^{\prime}\right\rangle=\int_{-\infty}^{\infty} p^{\prime}\left|\hat{\phi}_{s}\left(p^{\prime}\right)\right|^{2} \mathrm{~d} p^{\prime},\left\langle p^{\prime 2}\right\rangle=\int_{-\infty}^{\infty} p^{\prime 2}\left|\hat{\phi}_{s}\left(p^{\prime}\right)\right|^{2} \mathrm{~d} p^{\prime}
$$

which yield

$$
\left\langle p^{\prime}\right\rangle=16 A_{0}^{2} \eta \xi, \quad\left\langle p^{\prime 2}\right\rangle=\frac{32 \sqrt{2}}{3} A_{0}^{2} \eta^{3}+32 \sqrt{2} A_{0}^{2} \eta \xi^{3}
$$

The standard deviations of position

$$
\begin{aligned}
& \Delta x^{\prime \prime}=\sqrt{\left\langle x^{\prime 2}\right\rangle-\left\langle x^{\prime}\right\rangle^{2}} \text { and momentum } \\
& \Delta p^{\prime}=\sqrt{\left\langle p^{\prime 2}\right\rangle-\left\langle p^{\prime}\right\rangle^{2}} \text { are given by } \\
& \left(\Delta x^{\prime}\right)^{2}=A_{0}^{2}\left[\frac{\pi^{2}}{12 \eta}+4 \eta x_{0}^{\prime 2}\left(1-4 \sqrt{2} \eta A_{0}^{2}\right)\right]=\frac{\pi^{2}}{96 \eta^{2}}, \\
& \left(\Delta p^{\prime}\right)^{2}=32 \sqrt{2} A_{0}^{2}\left[\frac{1}{3} \eta^{3}+\eta \xi^{3}\left(1-4 \sqrt{2} \eta A_{0}^{2}\right)\right]=\frac{8}{3} \eta^{2},
\end{aligned}
$$

respectively. Thus Pang [27-29,47] obtain the uncertainty relation between position and momentum for the microscopic particle depicted by the nonlinear Schrödinger equation in Eq.15

$$
\Delta x^{\prime} \Delta p^{\prime}=\pi / 6
$$

This result is not related to the features of the microscopic particle (soliton) depicted by the nonlinear Schrödinger equation because Eq.59 has nothing to do with characteristic parameters of the nonlinear Schrödinger equation. $\pi$ in Eq.59 comes from of the integral coefficient $1 / \sqrt{2 \pi}$. For a quantized microscopic particle, $\pi$ in Eq.59 should be replaced by $\pi \hbar$, because Eq.52 is replaced by

$$
\phi_{s}(p, t)=\frac{1}{\sqrt{2 \pi \hbar}} \int_{-\infty}^{\infty} \mathrm{d} x \phi_{s}(x, t) \mathrm{e}^{-i p x / \hbar} .
$$

Thus the corresponding uncertainty relation of quantum microscopic particle is given by ${ }^{[24-27,47]}$

$$
\Delta x \Delta p=\pi \hbar / 6=h / 12
$$

This uncertainty principle also suggests that the position and momentum of the microscopic particle can be simultaneously determined in a certain degree. It is possible to estimate roughly the sizes of the uncertainty of these physical quantities. If it is required that $\phi_{\mathrm{s}}\left(x^{\prime}, \mathrm{t}^{\prime}\right)$ in Eq.22 or $\phi_{\mathrm{s}}\left(\mathrm{p}^{\prime}, \mathrm{t}^{\prime}\right)$ in Eq.53 satisfies the admissibility condition i.e., $\phi_{\mathrm{s}}(0) \approx 0$,we choose $\xi=140, \eta=\sqrt{300 / 0.253} / 2 \sqrt{2}$ and $\boldsymbol{x}_{0}^{\prime}=0$ in Eq.22 (In fact, in such a case we can get $\phi_{\mathrm{s}}(0) \approx 10^{-7}$, thus the admissibility condition can be satisfied). We then get $\Delta x^{\prime} \approx 0.02624$ and $\Delta p^{\prime} \approx 19.893$, according to Eqs.59 and 60. This result shows that the position and momentum of the microscopic particles in the nonlinear quantum mechanics could be determined simultaneously within a certain approximation, one of these cannot approach infinite.

Also, the uncertainty relation in Eq.61 or Eq.59 differ from the $\Delta x \Delta p>\hbar / 2$ in Eq.61 in the linear quantum mechanics. However, the minimum value $\Delta x \Delta p=\hbar / 2$ has not been obtained from both the solutions of linear Schrödinger equation and experimental measurement up to now, except for the coherent and squeezed states of 
microscopic particles. Therefore we can draw a conclusion that the minimum uncertainty relationship is a nonlinear effect, instead of linear effect, and a result of wave-corpuscle duality.

From this result we see that when the microscopic particles satisfy $\Delta x \Delta p>\hbar / 2$, then their motions obey laws of the linear quantum mechanics, the particles are some waves. When the uncertainty relationship of $\Delta x \Delta p=\hbar / 12$ or $\pi / 6$ is satisfied, the microscopic particles should be described by nonlinear quantum mechanics, and have a wave-corpuscle duality. If the position and momentum of the particles meets $\Delta x \Delta p=0$, then the particles have only a corpuscle feature, i.e., they are the classical particles. Therefore, the minimum uncertainty relation in Eqs.61 and 59 exhibits clearly the wave-corpuscle duality of microscopic particles described by nonlinear quantum mechanics, which bridges also the gap between the classical and linear quantum mechanics. This is a very interesting result in physics.

(3). The uncertainty relations of the coherent states

As a matter of fact, we can represent one-quantum coherent state of harmonic oscillator by ${ }^{[48]}$

$$
|\alpha\rangle=\exp \left(\alpha \hat{b}^{+}-\alpha^{*} \hat{b}\right)|0\rangle=\mathrm{e}^{-\alpha^{2} / 2} \sum_{n=0}^{\infty} \frac{\alpha^{n}}{\sqrt{n-1}} \hat{b}^{+n}|0\rangle,
$$

in the number picture, which is a coherent superposition of a large number of quanta. Thus

$$
\langle\alpha|\hat{x}| \alpha\rangle=\sqrt{\frac{\hbar}{2 \omega m}}\left(\alpha+\alpha^{*}\right),\langle\alpha|\hat{p}| \alpha\rangle=i \sqrt{\hbar m \omega}\left(\alpha-\alpha^{*}\right),
$$

and

$$
\begin{aligned}
& \left\langle\alpha\left|\hat{x}^{2}\right| \alpha\right\rangle=\frac{\hbar}{2 \omega m}\left(\alpha^{* 2}+\alpha^{2}+2 \alpha \alpha^{*}+1\right), \\
& \left\langle\alpha\left|\hat{p}^{2}\right| \alpha\right\rangle=\frac{\hbar \omega m}{2}\left(\alpha^{* 2}+\alpha^{2}-2 \alpha \alpha^{*}-1\right),
\end{aligned},
$$

where $\hat{x}=\sqrt{\frac{\hbar}{2 \omega m}}\left(\hat{b}+\hat{b}^{+}\right), \hat{\mathrm{p}}=\mathrm{i} \sqrt{\frac{\hbar \omega m}{2}}\left(\hat{b}^{+}-\hat{b}\right)$,

and $\hat{b}^{+}(\hat{b})$ is the creation (annihilation) operator of microscopic particle (quantum), $\alpha$ and $\alpha^{*}$ are some unknown functions, $\omega$ is the frequency of the particle, $m$ is its mass. Thus we can get

$$
(\Delta x)^{2}=\frac{\hbar}{2 \omega m},(\Delta \mathrm{p})^{2}=\frac{\hbar \omega \mathrm{m}}{2},\langle\Delta \mathrm{x}\rangle^{2}\langle\Delta \mathrm{p}\rangle^{2}=\frac{\hbar^{2}}{4}
$$

This is a minimum uncertainty relationship for the coherent state.

For the squeezed state of the microscopic particle: $|\beta\rangle=\exp \left[\beta\left(\mathrm{b}^{+2}-\mathrm{b}^{2}\right)\right]|0\rangle$, which is a two quanta coherent state, we can find that

$$
\left\langle\beta\left|\Delta x^{2}\right| \beta\right\rangle=\frac{\hbar}{2 \mathrm{~m} \omega} \mathrm{e}^{4 \beta},\left\langle\beta\left|\Delta \mathrm{p}^{2}\right| \beta\right\rangle=\frac{\hbar \mathrm{m} \omega}{2} \mathrm{e}^{-4 \beta},
$$

using a similar approach as the above. Here $\beta$ is the squeezed coefficient and $|\beta|<1$. Thus,

$$
\Delta x \Delta p=\frac{\hbar}{2}, \frac{\Delta x}{\Delta p}=\frac{1}{m \omega} \mathrm{e}^{8 \beta} \text {, or } \Delta p=\Delta x(\omega m) \mathrm{e}^{-8 \beta}
$$

This shows that the squeezed state meets a minimum uncertainty relationship, the momentum of the microscopic particle (quantum) is squeezed in the two-quanta coherent state compared to that in the one-quantum coherent state.

The above results show that both one-quantum and two-quanta coherent states satisfy the minimal uncertainty principle. This is the same with that of the microscopic particles in the nonlinear quantum mechanics. This means that coherent and squeezed states are a nonlinear quantum state, the coherence and squeezing of quanta are a kind of nonlinear quantum effect. Just so, the states of a microscopic particles described by the nonlinear Schrödinger Eq.8, such as the Davydov's wave functions [49], both $\mathrm{ID}_{1}>$ and $\mathrm{ID}_{2}>$, and Pang's wave function of exciton-solitons ${ }^{[50-53]}$ in protein molecules and acetanilide; the wave function of proton transfer in hydrogen-bonded systems and the BCS's wave function in superconductors [34], etc., are always represented by a coherent state. Hence, the coherence of particles does not belong to the systems described by linear quantum mechanics, because the coherent state cannot be obtained by superposition of linear waves, such as plane wave, de Broglie wave, or Bloch wave. Then the minimal uncertainty relation Eq.61, as well as Eqs.59 and 63, are only applicable to microscopic particles described by the nonlinear quantum mechanics. Thus it reflects the wave-corpuscle duality of the microscopic particles.

Also, the above results indicate not only the essences of nonlinear quantum effects of the coherent state or squeezing state but also that the minimal uncertainty relationship is an intrinsic feature of the nonlinear quantum mechanics systems including the coherent and squeezing states.

Pang et al. [50-53] also calculated the uncertainty relationship and quantum fluctuations and studied their properties in nonlinear electron-phonon systems based on the Holstein model by a new ansatz including the correlations among one-phonon coherent and two-phonon squeezing states and polaron state. Many interesting results were obtained, such as the minimum uncertainty relationship is related to the properties of the microscopic particles. The results enhanced the understanding of the significance and essences of the minimum uncertainty relationship. 


\section{CONCLUSIONS, RESOLUTION OF DIFFICULTIES OF LINEAR QUANTUM MECHANICS}

As it is known, the states and properties of microscopic particle were described by the linear Schrödinger Eq.3 in the quantum mechanics, but the microscopic particles have only a wave feature, not corpuscle feature as described in Introduction. In nonlinear quantum mechanics, we have broken through the hypothesis of independence of Hamiltonian operator of the systems on states of microscopic particles, forsaken the above linearity hypothesis of linear quantum mechanics and taken into account the true motions of each particle and background field and the interactions between them, thus the microscopic particles accepted a nonlinear interaction and their laws of motion are then described by Eqs.8-13 Thus natures and properties of the microscopic particles appear considerable changes, when compared with those in linear quantum mechanics. The changes can be summarized as follows [17-37].

1) In this new theory although the states of microscopic particles are still represented as a wave function $\phi(\boldsymbol{r}, t)$ in Eq.7, its absolute square,

$|\phi(\boldsymbol{r}, t)|^{2}=|\varphi(\boldsymbol{r}, t)|^{2}=\rho(\boldsymbol{r}, t)$, denotes no longer the probability of finding the microscopic particle at a given point in the space-time, and give just the mass density of the microscopic particles at that point. Thus we can find out the particle number or the mass of the particle from $\int_{-\infty}^{\infty}|\phi|^{2} \mathrm{~d} \tau=N$, the concept of probability is abandoned thoroughly in nonlinear quantum mechanics. Then the difficulty of statistical interpretation for the wave function of microscopic particle in quantum mechanics is solved.

2) The dynamic equations the particles satisfy are not the linear Schrödinger equation in Eq.2 and linear Klein-Gordon equation, but nonlinear Schrödinger equations in Eqs. 8 and 9 and nonlinear Klein-Gordon equations in Eqs.10 and 11. Their solutions have a wavecorpuscle duality, which is embedded by organic combination of envelope and carrier wave as shown in Figure 1. In such a case the particle has not only a wave feature, such as a certain amplitude, velocity, frequency, and wavevector, but also corpuscle natures, such as, a determinant mass centre, size, mass, momentum and energy. This is the first time to explain physically the wave- corpuscle duality of microscopic particles in quantum systems. This is a great advance of modern quantum theory, thus it solved a most great difficulty of one century existed in quantum mechanics.

3) In nonlinear quantum mechanics, $\int_{-\infty}^{\infty} \phi^{*} x \phi \mathrm{d} \tau$, $\frac{\partial}{\partial t} \int_{-\infty}^{\infty} \phi^{*} x \phi \mathrm{d} \tau$ and $\int \phi^{*} \widehat{H} \phi \mathrm{d} x$ or $\langle\phi|\widehat{H}| \phi\rangle$ are no longer some average values of the physical quantities in linear quantum mechanics, but represent the position, velocity and acceleration of the mass center and energy of the microscopic particles, respectively, and have determinant values. Thus, the presentations of physical quantities in the nonlinear quantum mechanics appear considerably the variations relative to those in linear quantum mechanics. This has solved the difficulty arising from the average values, which represent the physical quantities in linear quantum mechanics.

4) The microscopic particles have determinant mass, momentum and energy, and obey universal conservation laws of mass, momentum, energy and angular momentum. This amount to bridge over the gap between the classical mechanics and linear quantum mechanics.

5)The microscopic particles meet the classical collision rule, when they collide with each other. Although these particles are deformed in the collision, which denotes its wave feature, they can still retain their form and amplitude to move towards after collision, where a phase shift occurs only. This denotes that the microscopic particles in nonlinear quantum mechanics possess both corpuscle and wave property, but the corpuscle property differs from classical particles.

6) The position and momentum of the mass centre of microscopic particles are determinant, but their uncertainties obey only to a minimal uncertainty relation due to the wave-corpuscle duality, which differs from those in linear quantum mechanics. This means that the coordinate and momentum of microscopic particles may be simultaneously determined at a certain degree. This amount to bridge over the gap between the classical mechanics and linear quantum mechanics.

These show clearly the necessity, validity and importance of establishing nonlinear quantum mechanics. Thus the difficulties of linear quantum mechanics can be also solved thoroughly by nonlinear quantum mechanics. Therefore, to develop and to establish NLQM can solve problems disputed by scientists in the LQM field for about a century [7-9], can promote the development of physics and enhance and raise the knowledge and recognition levels to the essences of microscopic matter. We can predict that nonlinear quantum mechanics has extensive applications in physics, chemistry, biology, polymers, etc.

\section{ACKNOWLEDGEMENTS}

I would like to acknowledge the Major State Basic Research Development Program (973 program) of China for the financial support 
(grate No: 212011CB503 701).

\section{REFERENCES}

[1] Bohr, D. and Bub, J. (1966) A proposed solution of the measurement problem in quantum mechanics. Review of morden Physics, 6, 453-469.

doi:10.1103/RevModPhys.38.453

[2] Schrödinger, E. (1935) Die gegenwartige situation in der quantenmechanik, Naturwissenschaften, 23, 807-849. doi:10.1007/BF01491891

[3] Schrödinger, E. (1935) The present situation in quantum mechanics, a translation of translation of Schrodinger. Proceedings of the American Philosophical Society, 124, 323-338.

[4] Schrödinger, E. (1926) An undulatory theory of the mechanics of atoms and molecules. Physical Review, 28, 1049-1070. doi:10.1103/PhysRev.28.1049

[5] Heisenberg, W. Z. (1925) Über die quantentheoretische umdeu- tung kinematischer und mechanischer beziehungen. Zeitschrift der Physik, 33, 879-893. doi:10.1007/BF01328377

[6] Heisenberg, W. and Euler, H. (1936) Folgerungen aus der Diracschen Theorie des Positrons. Physics and Astronomy, 98, 714-732. doi:10.1007/BF01343663

[7] Born, M. and Infeld, L. (1934) Foundations of the New Field Theory, Proceedings of the American Philosophical Society, 144, 425.

[8] Dirac, P.A.M. (1948) Quantum Theory of Localizable Dynamical Systems, Physical Review, 73, 1092. doi:10.1103/PhysRev.73.1092

[9] Diner, S., Farque, D., Lochak, G., and Selleri, F. (1984) The wave-particle dualism. Riedel, Dordrecht.

[10] Ferrero, M. and Van der Merwe, A. (1997) New developments on fundamental problems in quantum physics. Kluwer, Dordrecht.

[11] Ferrero, M. and Van der Merwe, A. (1995) Fundamental problems in quantum physics. Kluwer, Dordrecht.

[12] de Broglie, L., (1960) Nonlinear wave mechanics: A causal interpretation, Elsevier, Amsterdam.

[13] de Broglie, L., (1955) Une interpretation nouvelle de la mechanique ondulatoire: Est-elle possible? Nuovo Cimento, 1, 37-50.

[14] Bohm, D.A. (1952) Suggested interpretation of the quantum theory in terms of 'hidden' variables. Physical Review, 85, 166-180.

[15] Potter, J. (1973) Quantum mechanics. North-Holland publishing Co. Amsterdam.

[16] Jammer, M. (1989) The concettual development of quantum mechanics. Tomash Publishers, Los Angeles.

[17] Einstein, A., Podolsky, B. and Rosen, N. (1935) The appearance of this work motivated the present-shall I say lecture or general confession? Physical. Review, 47, 777-780. doi:10.1103/PhysRev.47.777

[18] Einstein, A.P., (1979) A centenary Volume. Harvard University Press, Cambridge.

[19] Pang, X.F. (1985) Problems of nonlinear quantum mechanics. Sichuan Normal University Press, Chengdu.

[20] Pang, X.F. (2008) The Schrodinger equation only descrybes approximately the properties of motion of microscopic particles in quantum mechanics. Nature Sciences,
3, 29.

[21] Pang, X.F. (1985) The fundamental principles and theory of nonlinear quantum mechanics. China Journal of Potential Science, 5, 16.

[22] Pang, X.F. (1982) Macroscopic quantum mechanics. China Nature Journal, 4, 254.

[23] Pang, X.F. (1986) Bose-condensed properties in supercon-ducors. Journal of Science Exploration, 4, 70.

[24] Pang, X.F. (1991) The theory of nonlinear quantum mechanics: In research of new sciences, Science and Techbology Press, Hunan, 16-20.

[25] Pang, X.F. (2008) The wave-corpuscle duality of microscopic particles depicted by nonlinear Schrodinger equation. Physica B, 403, 4292-4300. doi:10.1016/j.physb.2008.09.031

[26] Pang, X.F. (2008) Features and states of microscopic particles in nonlinear quantum-mechanics systems. Frontiers of physics in China, 3, 413.

[27] Pang, X.F. (2005) Quantum mechanics in nonlinear systems. World Scientific Publishing Co., Singapore. doi:10.1142/9789812567789

[28] Pang, X.F. (2009) Nonlinear quantum mechanics. China Electronic Industry Press, Beijing.

[29] Pang, X.F. (1994) The Theory of nonlinear quantum mechanics. Chinese Chongqing Press, Chongqing.

[30] Pang, X.F. (2006) Establishment of nonlinear quantum mechanics. Research and Development and of World Science and Technology, 28, 11.

[31] Pang, X.F. (2003) Rules of motion of microscopic particles in nonlinear systems. Research and Development and of World Science and Technology, 24, 54.

[32] Pang, X.F. (2006) Features of motion of microscopic particles in nonlinear systems and nonlinear quantum mechanics in sciencetific proceding-physics and others. Atomic Energy Press, Beijing.

[33] Parks, R. D. (1969) Superconductivity. Marcel, Dekker.

[34] Josephson, D.A. (1965), Supercurrents through barriers, Advanced Physics, 14, 39-451.

[35] Suint-James, D. et al., (1966) Type-II superconductivity, Pergamon, Oxford.

[36] Bardeen, L.N., Cooper L.N. and Schrieffer, J. R. (1957) Superconductivity theory. Physical Review, 108, 11751204. doi:10.1103/PhysRev.108.1175

[37] Barenghi, C.F., Donnerlly, R.J. and Vinen, W.F. (2001) Quantized vortex dynamics and superfluid turbulence. Springer, Berlin. doi:10.1007/3-540-45542-6

[38] Donnely, R.J. (1991) Quantum vortices in heliem II. Cambridge University Press, Cambridge.

[39] Pang, X.F. (2003) Soliton physics. Sichuan Science and Technology Press, Chengdu.

[40] Guo, B.L. and Pang, X.F. (1987) Solitons. Chinese Science Press, Beijing.

[41] Zakharov, V.E. and Shabat, A.B. (1972) Exact theory of two-dimensional self-focusing and one-dimensional selfdomulation of wave in nonlinear media. Soviet Physics JETP, 34, 62.

[42] Zakharov, V.E. and Shabat, A.B. (1973) Interaction between solitons in a stable medium. Soviet Physics JETP, 37, 823

[43] Lax, P.D. (1992) Integrals of nonlinear equations of evolution and solitary waves, Cambridge University Press, Cambridge, 107-351 
[44] Pang, X.F. (2010) Collision properties of microscopic particles described by nonlinear Schrodinger equation. International Journal of Nonlinear science and numerical Simulation, 11, 1069-1075.

[45] Stiefel, J. (1965) Einfuhrung in die numerische mathematik. Teubner Verlag, Stuttgart.

[46] Atkinson, K.E. (1987) An Introdution to numerical analysis. Wiley, New York.

[47] Pang, X.F. (2009) Uncertainty features of microscopic particles described by nonlinear SchrÖdinger equation. Physica B, 405, 4327-4331. doi:10.1016/j.physb.2009.08.027

[48] Glanber, R.J. (1963) Coherent and incoherent states of the radiation field. Physical Review, 13, 2766-2788. doi:10.1103/PhysRev.131.2766

[49] Davydov, A. S. (1985) Solitons in molecular systems. D.
Reidel Publishing, Dordrecht.

[50] Pang, X.F. (2008) Properties of nonadiabatic quantum fluctuations for the strongly coupled electron-phonon system. Science in China Series G, 51, 225-336.

[51] Pang, X.F. (1999) Influence of the soliton in anharmonic molecular crystals with temperature on Mossbauer effect. European Physical Journal B, 10, 415. doi:10.1007/s100510050871

[52] Pang, X.F. (2001) The lifetime of the soliton in the improved Davydov model at the biological temperature $300 \mathrm{~K}$ for protein molecules. Physics and Astronomy, 19, 297-316. doi:10.1007/s100510170339

[53] Pang, X.F. (1990) The properties of collective excitation in organic protein molecular system. Journal of Physics: Condensed Matter, 2, 9541. doi:10.1088/0953-8984/2/48/008 